\title{
86421 - ENVELHECIMENTO SAUDÁVEL: UMA REVISÃO INTEGRATIVA
}

\author{
Pôster - Gerontologia
}

Marcia Rejane Estima Pedone / Pedone, MRE / PUCRS

O processo de envelhecimento e suas modificações vêm sendo estudados há muito tempo. Até o século XX, os idosos eram estereotipados, além de haver muitos mitos acerca desses indivíduos. No decorrer do século diversos, autores foram observando e analisando que o envelhecimento era um processo heterogêneo, assim passou-se a pesquisar os fatores para um envelhecimento saudável (CUPERTINO; ROSA \& RIBEIRO, 2007). Rowe e Kahn (1998) foram os primeiros autores que propuseram as possíveis trajetórias no envelhecimento humano (normal, patológico e saudável), sendo que a definição descrita por eles foi ter um baixo risco de doenças e de incapacidades funcionais relacionadas às doenças; alto funcionamento mental e físico; e envolvimento ativo com a vida. $\mathrm{O}$ envelhecimento bem-sucedido é afetado tanto por influências precoces, por condições favoráveis na infância que melhoram significativamente os escores de envelhecimento saudável, quanto pela educação, renda, comportamentos de saúde, apoio social, e religiosidade. Sugere-se que se exercitar, manter um peso adequado e não fumar provavelmente podem se traduzir em um envelhecimento mais saudável. Os autores trazem os termos "envelhecimento saudável" e "envelhecimento bem-sucedido" como termos intercambiáveis no seu estudo (FERDOWS; JENSEN \& TARRAF, 2018). Objetivo: revisar as concepções na literatura científica acerca do envelhecimento saudável.Trata-se de uma revisão integrativa para buscar estudos que apresentavam a concepção de Envelhecimento Saudável. Os critérios de inclusão foram: ter o texto completo disponível, publicados nos últimos 5 anos, sendo a última data da pesquisa: 17/06/2019; Os critérios de exclusão foram: artigos de revisão, artigos que não abordavam o assunto pesquisado e artigos que não apresentavam a concepção de Envelhecimento Saudável.Para estratégia de busca eletrônica foi consultado a base de dados PubMed. Três conjuntos de termos foram cruzados para a busca eletrônica: "Healthy Aging" AND intervention AND population. A pesquisa resultou em 314 artigos, cujos dados foram inseridos em uma planilha previamente construída com os itens de interesse. Também serão apresentados possíveis vieses encontrados em relação à análise realizada.a revisão integrativa, segundo Ercole, Melo e Alcoforado (2014). 